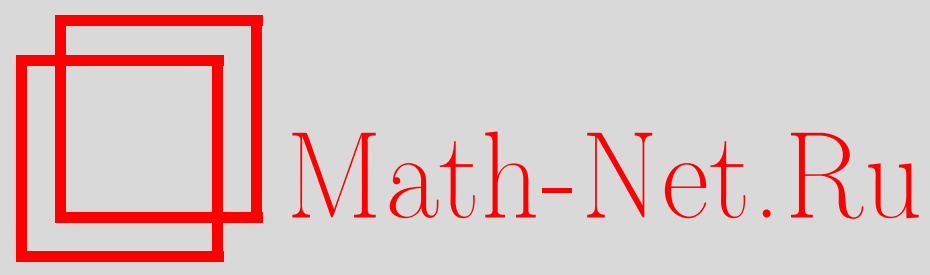

Т. В. Азарнова, Оценки элементов обратных матриц для одного класса операторов с матрицами специальной структуры, Матем. заметки, 2002, том 72, выпуск 1, 3-10

DOI: https://doi.org/10.4213/mzm399

Использование Общероссийского математического портала Math-Net.Ru подразумевает, что вы прочитали и согласны с пользовательским соглашением http://www . mathnet.ru/rus/agreement

Параметры загрузки:

IP: 35.173 .137 .237

26 апреля 2023 г., 04:34:03

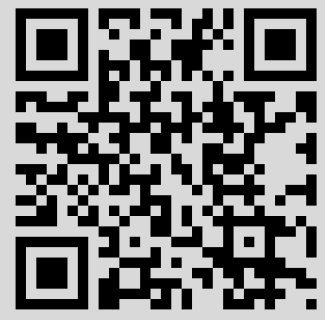




\section{ОЦЕНКИ ЭЛЕМЕНТОВ ОБРАТНЫХ МАТРИЦ ДЛЯ ОДНОГО КЛАССА ОПЕРАТОРОВ С МАТРИЦАМИ СПЕЦИАЛЬНОЙ СТРУКТУРЫ}

\section{Т. В. Азарнова}

В статье рассматриваются вопросы, связанные со структурой обратных матриц линейных ограниченных операторов, действующих в бесконечномерных комплексных банаховых пространствах. Получены конкретные оценки элементов обратных матриц для ограниченных операторов, матрицы которых имеют специальную структуру. Матрица вводится как специальная операторнозначная функция, заданная на индексном множестве. Структура матрицы описывается поведением данной функции на элементах специального разбиения индексного множества. Метод получения оценок построен на основе анализа рядов Фурье некоторых сильно непрерьвных периодических функций.

Библиография: 9 названий.

При решении задач, возникающих в вычислительной математике, в теории интегральных операторов и теории функций, часто используется матричный анализ линейных ограниченных операторов. В частности, существенное применение, особенно в вычислительной математике, находят оценки элементов обратных матриц. Наиболее изученными являются оценки элементов обратных матриц операторов, действующих в конечномерных пространствах. Исследованию оценок элементов обратных матриц для операторов, действующих в бесконечномерных пространствах, также посвящен ряд работ [1]-[7]. Однако для случая бесконечномерных пространств конкретные оценки элементов обратных матриц (оценки, которые непосредственно можно вычислить по характеристикам исходного оператора) получены для небольшого числа классов операторов. В основном это операторы с разреженными или псевдоразреженными матрицами, структура которых эффективно описьвается через оценки коэффициентов матрищы $A_{i j}$, $(i, j) \in \mathbb{Z} \times \mathbb{Z}$, расположенных на элементах диагонального разбиения множества $\mathbb{Z} \times \mathbb{Z}$, т.е. разбиения $\left\{S_{k}: k \in \mathbb{Z}\right\}$ вида

$$
S_{k}=\{(i, j) \in \mathbb{Z} \times \mathbb{Z}: i-j=k\} .
$$

Поскольку для псевдоразреженных матриц большинство коэффициентов являются малыми по норме, то оценки коэффициентов матрицы, расположенных на элементах диагонального разбиения, обычно характеризуют закон убывания внедиагональных элементов матрицы.

Работа выполнена при финансовой поддержке Российского фонда фундаментальных исследований, грант № 01-01-00408. 
Актуальность новых исследований связана с изучением новых классов операторов или с получением более качественных оценок. Введение в рассмотрение новых классов операторов диктуется различными приложениями. Возникают операторы с новой структурой матриц, расширяется круг пространств, в которых действуют операторы.

В данной статье изучаются действующие в абстрактном бесконечномерном комплексном банаховом пространстве $X$ операторы, структуру матрищы которых эффективно описьвать через оценки коэффициентов $A_{i j},(i, j) \in \mathbb{Z} \times \mathbb{Z}$, расположенных на элементах специального (недиагонального) разбиения множества $\mathbb{Z} \times \mathbb{Z}$. Для пространства $l_{2}$ аналогичньй с точки зрения структуры матрицы класс операторов рассматривался в работе И. А. Блатова [1]. При получении оценок, характеризующих структуру обратной матрицы изучаемого оператора $A$, им использовалась техника полиномов Чебышева, наименее уклоняюшихся от $1 / x$ на $\sigma(A)$. Такой подход позволяет получать только асимптотические оценки. В данной работе мы расширяем круг пространств, в которых действуют операторы, и получаем не асимптотические, а конкретные оценки элементов обратных матрищ. Конкретные оценки представляют больший интерес с прикладной точки зрения, поскольку их можно вычислить по характеристикам исходного оператора. Используемый в работе аппарат получения конкретных оценок является обобщением на случай недиагонального разбиения техники получения конкретных оценок, разработанной в работе А. Г. Баскакова [2].

Пусть End $X$ - банахова алгебра линейных ограниченных операторов, действующих в бесконечномерном комплексном банаховом пространстве $X$. Введем матричное представление операторов из банаховой алгебры End $X$. Для этого предположим, что в $\mathrm{X}$ есть дизъюнктная последовательность проекторов $\mathscr{P}=\left\{P_{j} \in\right.$ End $\left.X: j \in \mathbb{Z}\right\}$, обладающая следующими свойствами:

1. ряд $\sum_{k \in \mathbb{Z}} P_{k} x$ безусловно сходится к $x$ для любого $x \in X$;

2. $\sup _{\alpha_{k} \in C,\left|\alpha_{k}\right|=1}\left\|\sum_{k \in \mathbb{Z}} \alpha_{k} P_{k}\right\|=1$ (из предположения 1 в общем случае следует конечность данной величины);

3. для любых конечных подмножеств $\sigma_{1}, \sigma_{2}, \Delta_{1}, \Delta_{2}$ из множества $\mathbb{Z}$, обладающих свойствами $\sigma_{1} \cap \sigma_{2}=\varnothing, \Delta_{1} \cap \Delta_{2}=\varnothing$, и для любого оператора $A$ из End $X$ имеет место равенство

$$
\begin{aligned}
& \left\|\sum_{i \in \sigma_{1}, j \in \Delta_{1}} P_{i} A P_{j}+\sum_{i \in \sigma_{2}, j \in \Delta_{2}} P_{i} A P_{j}\right\| \\
& \quad=\max \left\{\left\|\sum_{i \in \sigma_{1}, j \in \Delta_{1}} P_{i} A P_{j}\right\|,\left\|\sum_{i \in \sigma_{2}, j \in \Delta_{2}} P_{i} A P_{j}\right\|\right\} .
\end{aligned}
$$

В дальнейшем такую последовательность проекторов $\mathscr{P}$ будем называть разложением единииы.

$\mathrm{C}$ помощью данной дизъюнктной системы проекторов определим матрищу $\mathscr{A}: \mathbb{Z} \times \mathbb{Z} \rightarrow$ End $X$ следующим образом:

$$
\mathscr{A}(i, j)=A_{i j}=P_{i} A P_{j} \quad((i, j) \in \mathbb{Z} \times \mathbb{Z}) .
$$

В качестве примера рассмотрим построение матриц операторов, действуюших в пространствах $X=L_{p q}=L_{p q}(\mathbb{R}, E) \quad(1 \leqslant q \leqslant \infty, p=0$ или $1 \leqslant p<\infty)(E-$ конечномерное комплексное банахово пространство), состоящих из классов измеримых совпадающих почти всюду функций $x: \mathbb{R} \rightarrow E$ таких, что при любом $k \in \mathbb{Z}$ функция $x_{k}:[0,1] \rightarrow E$, 
$x_{k}(t)=x(t+k)$ принадлежит пространству $L_{q}=L_{q}([0,1], E)$, а семейство функций $x_{k}(k \in \mathbb{Z})$ принадлежит пространству последовательностей $l_{p}\left(\mathbb{Z}, L_{q}([0,1], E)\right)$. В качестве разложения единищы $\mathscr{P}$ возьмем дизъюнктную последовательность проекторов $P_{k}: L_{p q} \rightarrow L_{p q}, k \in \mathbb{Z}$, вида

$$
P_{k} x=\chi_{k} x
$$

где $\chi_{k}$ - характеристическая функция множества $H_{k}=\{t \in \mathbb{R}: t=k+h, h \in[0,1]\}$.

Легко проверить, что эта система проекторов удовлетворяет приведенным выше трем свойствам для разложения единицы, а операторы

$$
A_{i j} x=P_{i} A P_{j} x=\chi_{i}\left(A\left(\chi_{j} x\right)\right)
$$

вполне однозначно определяются операторами $a_{i j}: L_{q} \rightarrow L_{q}$ вида

$$
a_{i j} x=z, \quad z(t)=A\left(\chi_{j} \widetilde{x}\right)(t+i), \quad t \in[0,1]
$$

где функция $\widetilde{x}: \mathbb{R} \rightarrow E$ строится по функции $x$ следуюшим образом:

$$
\widetilde{x}(t)= \begin{cases}x(t), & t \in H_{j}, \\ 0, & t \notin H_{j} .\end{cases}
$$

При этом $\left\|a_{i j}\right\|_{\text {End } L_{q}}=\left\|A_{i j}\right\|_{\text {End } L_{p q}}$. Таким образом, в данном случае в качестве матрицы оператора можно рассматривать матрицу с элементами $a_{i j}: L_{q} \rightarrow L_{q}$. Полученное матричное представление операторов в пространствах $L_{p q}$ находит эффективное применение при решении ряда задач. В частности, с помощью матричного представления в работе [8] доказаны эффективные условия обратимости специальных классов интегральных операторов, а в [9] получены оценки для ядер операторов, обратных к интегральным.

Обоснование корректности матричного представления (1) можно найти в работах [2], [6]. Так же, как и для обычных матриц, для таким образом введенных матриц можно ввести понятие диагональной матрищы и обосновать некоторое представление матрицы произвольного оператора $A \in$ End $X$ суммой диагональных матриц. С этой целью в рассмотрение вводится сильно непрерьвное изометрическое представление $P$ из группы $\mathbb{T}=\{\theta \in \mathbb{C}:|\theta|=1\}$ в End $X$

$$
P(\theta) x=\sum_{k \in \mathbb{Z}} P_{k} \theta^{k} x, \quad \theta \in \mathbb{T},
$$

и по оператору $A$ с помощью данного представления строится сильно непрерьвная периодическая функция $\Phi_{A}: \mathbb{T} \rightarrow$ End $X$,

$$
\Phi_{A}(\theta)=P(\theta) A P\left(\theta^{-1}\right), \quad\left\|\Phi_{A}(\theta)\right\|=\|A\| .
$$

Ряд Фурье данной функции

$$
\Phi_{A}(\theta) \sim \sum_{k \in \mathbb{Z}} A_{k} \theta^{k}
$$


сходится по Чезаро, а его коэффициенты $A_{k}$ представимы в виде сильно сходящихся рядов

$$
A_{k} \sim \sum_{i=j=k} A_{i j}
$$

причем $\left\|A_{k}\right\|=\sup _{|i-j|=k} A_{i j}$. Матрищы операторов $A_{k}$ являются диагональньми, т.е. могут иметь ненулевые элементы лишь на одной $k$-й диагонали (понятие диагонали аналогично понятию диагонали для обычных матриц), совпадающей с $k$-й диагональю матрицы оператора А.

Ряд

$$
A=\Phi_{A}(1) \sim \sum_{k \in \mathbb{Z}} A_{k}
$$

будем назьвать

Обозначим исследуемьй в данной статье класс операторов $\operatorname{End}(X, S, \gamma)$. Для описания данного класса операторов введем специальное разбиение $\left\{S_{k}\right\}$ множества $\mathbb{Z} \times \mathbb{Z}$. Разбиение вводится через, так назьваемое, порождающее множество $S \subseteq \mathbb{Z} \times \mathbb{Z}$,

$$
S=\left\{(i, j) \in \mathbb{Z} \times \mathbb{Z}:|i-j|=n_{q}, q=1,2, \ldots, l\right\}
$$

$\left(n_{q}, q=1,2, \ldots, l,-\right.$ натуральные числа, одно из которых обязательно равно 0), и порождающую матрищу $W=\left(W_{i j}\right)$,

$$
W_{i j}= \begin{cases}1, & (i, j) \in S, \\ 0, & (i, j) \notin S\end{cases}
$$

$\left(l\right.$ - количество диагоналей, $n_{q}, q=1,2, \ldots, l,-$ номера диагоналей). По матрице $W$ построим разбиение

$$
\begin{gathered}
S_{1}=S, \quad S_{k}=\operatorname{supp} W^{k} \backslash \operatorname{supp} W^{k-1}, \quad k \geqslant 2, \quad k \in \mathbb{N}, \\
S_{\infty}=\left\{(i, j) \in \mathbb{Z} \times \mathbb{Z}:(i, j) \notin S_{k} \forall k \in \mathbb{N}\right\}=(\mathbb{Z} \times \mathbb{Z}) \backslash \bigcup_{k \in \mathbb{N}} S_{k},
\end{gathered}
$$

где $\operatorname{supp} W^{k}$ - носитель матрицы $W^{k}=W * W * \cdots * W$,

$$
\operatorname{supp} W^{k}=\left\{(i, j) \in \mathbb{Z} \times \mathbb{Z}: W^{k}(i, j) \neq 0\right\} .
$$

Легко показать, что

$$
\operatorname{supp} W^{k} \subseteq\left\{(i, j) \in \mathbb{Z} \times \mathbb{Z}:|i-j|=\beta_{1} n_{1}+\cdots+\beta_{l} n_{l}\right\}, \quad k \geqslant 2,
$$

где $\beta_{q}, q=1, \ldots, l,-$ целые числа такие, что $\sum_{q=1}^{l}\left|\beta_{q}\right| \leqslant k$.

Заметим, что при $q=1,2$ и $n_{1}=0, n_{2}=1$ полученное разбиение множества $\mathbb{Z} \times \mathbb{Z}$ является диагональным.

Оператор $A \in$ End $X$ отнесем к классу $\operatorname{End}(X, S, \gamma)$, если существуют $\gamma=\gamma(A) \in$ $(0,1), M=M(A)>0$ такие, что верны оценки

$$
\sup _{(i, j) \in S_{k}}\left\|A_{i j}\right\| \leqslant M \gamma^{|k|}, \quad k \in \mathbb{N} .
$$


Для операторов данного класса ряд Фурье введенной ранее функции $\Phi_{A}: \mathbb{T} \rightarrow$ End $X$,

$$
\Phi_{A}(\theta)=\sum_{k \in \mathbb{Z}} A_{k} \theta^{k}, \quad \theta \in \mathbb{T},
$$

сходится абсолютно. Найдем некоторую область комплексной плоскости, на которую функция $\Phi_{A}$ допускает голоморфное продолжение

$$
\Phi_{A}(z)=\sum_{k \in \mathbb{Z}} A_{k} z^{k} .
$$

Чтобы найти данную область, проведем следующую последовательность оценок:

$$
\left\|\sum_{k \in \mathbb{Z}} A_{k} z^{k}\right\| \leqslant \sum_{k \geqslant 1} M \gamma^{k} \sum_{\left|\beta_{1}\right|+\cdots+\left|\beta_{l}\right|=k}|z|^{\beta_{1} n_{1}+\cdots+\beta_{l} n_{l}} .
$$

Для случая $|z|>1$ дальнейшую оценку можно продолжить в виде

$$
\begin{aligned}
\left\|\sum_{k \in \mathbb{Z}} A_{k} z^{k}\right\| & \leqslant \sum_{k \geqslant 1} M \gamma^{k} 2^{l} \frac{(k+l-1) !}{k !(l-1) !}|z|^{k n_{l}} \\
& =\frac{M 2^{l}}{(l-1) !} \sum_{k \geqslant 1}\left(\gamma|z|^{n_{l}}\right)^{k}(k+l-1) \cdots(k+1) \\
& =\frac{M 2^{l}}{(l-1) !}\left(\sum_{k \geqslant 1}\left(\gamma|z|^{n_{l}}\right)^{k+l-1}\right)^{(l-1)}
\end{aligned}
$$

а для случая $|z|<1$ в виде

$$
\begin{aligned}
\left\|\sum_{k \in Z} A_{k} z^{k}\right\| & \leqslant \sum_{k \geqslant 1} M \gamma^{k} 2^{l} \frac{(k+l-1) !}{k !(l-1) !}|z|^{-k n_{l}} \\
& =\frac{M 2^{l}}{(l-1) !} \sum_{k \geqslant 1}\left(\gamma|z|^{-n_{l}}\right)^{k}(k+l-1) \cdots(k+1) \\
& =\frac{M 2^{l}}{(l-1) !}\left(\sum_{k \geqslant 1}\left(\gamma|z|^{-n_{l}}\right)^{k+l-1}\right)^{(l-1)} .
\end{aligned}
$$

Полученные оценки показывают, что функция $\Phi_{A}: \mathbb{T} \rightarrow \operatorname{End} X$ допускает голоморфное продолжение на кольцо

$$
K=\left\{z \in C: \sqrt[n_{l}]{\gamma}<|z|<\sqrt[n_{l}]{\frac{1}{\gamma}}\right\}
$$

Предположим, что оператор $A$ обратим; тогда значения функции $\Phi_{A}$ также обратимы на $\mathbb{T}$ и

$$
\Phi^{-1}(\theta)=P(\theta) A^{-1} P\left(\theta^{-1}\right)=\Phi_{A^{-1}}(\theta), \quad\left\|\Phi_{A}^{-1}(\theta)\right\|=\left\|A^{-1}\right\| .
$$


Чтобы для оценки диагоналей матрищы обратного оператора $A^{-1}$ воспользоваться техникой рядов Лорана, найдем окрестность $\mathbb{T}$ в виде кольца, в котором значения голомор фного продолжения $\Phi_{A}: K \rightarrow \operatorname{End} X$ обратимы. В частности, $\Phi_{A}(z)$ будет обратимо для $z \in \mathbb{C}$, которые удовлетворяют следующему неравенству:

$$
\left\|\Phi_{A}(z)-\Phi_{A}(\theta)\right\| \leqslant \frac{1}{2}
$$

Оценим сверху левую часть данного неравенства:

$$
\begin{aligned}
\left\|\Phi_{A}(z)-\Phi_{A}(\theta)\right\| & =\left\|\sum_{k \in \mathbb{Z}} A_{k} z^{k}-\sum_{k \in \mathbb{Z}} A_{k} \theta^{k}\right\| \\
& \leqslant\left\|\sum_{k \in \mathbb{Z}} A_{k}\left(z^{k}-\theta^{k}\right)\right\| \leqslant \sum_{k \in \mathbb{Z}}\left\|A_{k}\right\|\left|z^{k}-\theta^{k}\right| \\
& \leqslant \sum_{k \in \mathbb{Z}}\left\|A_{k}\right\||| z^{k}\left|\theta^{k}-\right| \theta^{k}\left|\theta^{k}\right|=\sum_{k \in \mathbb{Z}}\left\|A_{k}\right\| \| z^{k}|-1| \\
& \leqslant\left.\sum_{k \geqslant 1} M \gamma^{k} \sum_{\left|\beta_{1}\right|+\cdots+\left|\beta_{l}\right|=k}|| z\right|^{\beta_{1} n_{1}+\cdots+\beta_{l} n_{l}-1 \mid .}
\end{aligned}
$$

Теперь проанализируем отдельно два случая: 1) $|z|>1$ и 2) $|z|<1$.

Для $|z|>1$ имеем

$$
\begin{aligned}
\left\|\Phi_{A}(z)-\Phi_{A}(\theta)\right\| \leqslant & \sum_{k \geqslant 1} M \gamma^{k} 2^{l} \frac{(k+l-1) !}{k !(l-1) !}\left(|z|^{k n_{l}}-1\right) \\
= & \frac{2^{l} M}{(l-1) !}\left[\sum_{k \geqslant 1} \gamma^{k} \frac{(k+l-1) !}{k !}|z|^{k n_{l}}-\sum_{k \geqslant 1} \gamma^{k} \frac{(k+l-1) !}{k !}\right] \\
= & \frac{2^{l} M}{(l-1) !}\left[\sum_{k \geqslant 1}\left(\gamma|z|^{n_{l}}\right)^{k}(k+1)(k+2) \cdots(k+l-1)\right. \\
& \left.-\sum_{k \geqslant 1} \gamma^{k}(k+1)(k+2) \cdots(k+l-1)\right] .
\end{aligned}
$$

Обозначим

$$
x=\gamma|z|^{n_{l}}, \quad y=\gamma
$$

и продолжим оценку:

$$
\begin{aligned}
\left\|\Phi_{A}(z)-\Phi_{A}(\theta)\right\| \leqslant & \frac{2^{l} M}{(l-1) !}\left(\sum_{k \geqslant 1} x^{k}(k+1)(k+2) \cdots(k+l-1)\right. \\
& \left.-\sum_{k \geqslant 1} y^{k}(k+1)(k+2) \cdots(k+l-1)\right) \\
= & \frac{2^{l} M}{(l-1) !}\left(\left(\sum_{k \geqslant 1} x^{k+l-1}\right)^{(l-1)}-\left(\sum_{k \geqslant 1} y^{k+l-1}\right)^{(l-1)}\right) \\
= & \frac{2^{l} M}{(l-1) !}\left(\left(\frac{x^{l}}{1-x}\right)^{(l-1)}-\left(\frac{y^{l}}{1-y}\right)^{(l-1)}\right)
\end{aligned}
$$




$$
\begin{aligned}
& =\frac{2^{l} M}{(l-1) !}\left((l-1) !\left(-1+\frac{1}{(1-x)^{l}}\right)-(l-1) !\left(-1+\frac{1}{(1-y)^{l}}\right)\right) \\
& =2^{l} M\left(\frac{1}{(1-x)^{l}}-\frac{1}{(1-y)^{l}}\right) .
\end{aligned}
$$

Закончим оценку левой части неравенства (2) для случая $|z|>1$ и продолжим его решение с учетом полученной оценки:

$$
\begin{gathered}
\frac{1}{(1-x)^{l}}-\frac{1}{(1-y)^{l}} \leqslant \frac{1}{2^{l+1} M\left\|A^{-1}\right\|}, \\
\frac{1}{(1-x)^{l}} \leqslant \frac{1}{2^{l+1} M\left\|A^{-1}\right\|}+\frac{1}{(1-y)^{l}}=\frac{(1-y)^{l}+2^{l+1} M\left\|A^{-1}\right\|}{2^{l+1} M\left\|A^{-1}\right\|(1-y)^{l}}, \\
(1-x)^{l} \geqslant \frac{2^{l+1} M\left\|A^{-1}\right\|(1-y)^{l}}{(1-y)^{l}+2^{l+1} M\left\|A^{-1}\right\|}, \quad(1-x) \geqslant \sqrt[l]{\frac{2^{l+1} M\left\|A^{-1}\right\|(1-y)^{l}}{(1-\gamma)^{l}+2^{l+1} M\left\|A^{-1}\right\|}} \\
x \leqslant 1-\sqrt[l]{\frac{2^{l+1} M\left\|A^{-1}\right\|(1-y)^{l}}{(1-y)^{l}+2^{l+1} M\left\|A^{-1}\right\|}} .
\end{gathered}
$$

Вернемся к старым обозначениям. Тогда

$$
\begin{aligned}
& \gamma|z|^{n_{l}} \leqslant 1-\sqrt[l]{\frac{2^{l+1} M\left\|A^{-1}\right\|(1-\gamma)^{l}}{(1-\gamma)^{l}+2^{l+1} M\left\|A^{-1}\right\|}}, \\
&|z| \leqslant \sqrt[n_{l}]{\frac{1}{\gamma}-\sqrt[l]{\frac{2^{l+1} M\left\|A^{-1}\right\|(1-\gamma)^{l}}{\left(\gamma-\gamma^{2}\right)^{l}+2^{l+1} \gamma^{l} M\left\|A^{-1}\right\|}}}
\end{aligned}
$$

Проводя аналогичные рассуждения для $|z|<1$, получим оценки

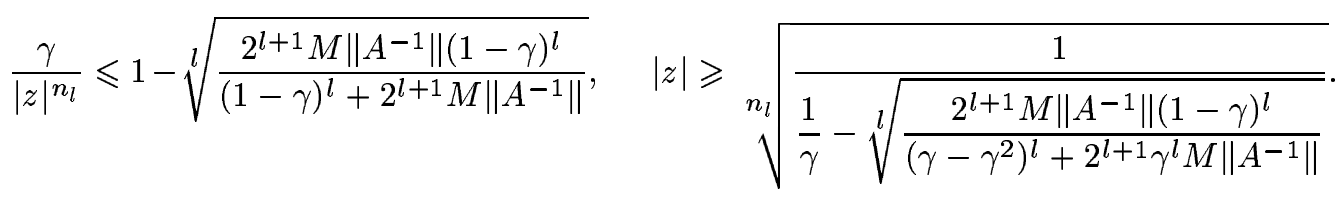

Обозначим

$$
\rho=\sqrt[n_{l}]{\frac{\gamma}{1-\sqrt[l]{\frac{2^{l+1} M\left\|A^{-1}\right\|(1-\gamma)^{l}}{(1-\gamma)^{l}+2^{l+1} M\left\|A^{-1}\right\|}}}}
$$

Таким образом, в кольце

$$
K_{1}(\rho)=\left\{z \in C: \rho \leqslant|z| \leqslant \frac{1}{\rho}\right\}
$$

голоморфное продолжение $\Phi_{A}(z)$ имеет обратимые значения. В данном кольце функция $\Phi_{A^{-1}}(z)=\Phi_{A}^{-1}(z)$ голоморфна и, следовательно, может быть представлена в виде ряда Лорана

$$
\Phi_{A^{-1}}(z)=\sum_{k \in Z} B_{k} z^{k}, \quad B_{k}=\sum_{i-j=k} B_{i j}, \quad\left\|B_{k}\right\|=\sup _{i-j=k} B_{i j} .
$$


Коэффициенты $B_{k}, k \neq 0$, вычисляются по формуле

$$
B_{k}=\frac{1}{2 \pi i} \int_{\Gamma} \frac{\Phi_{A^{-1}}(\xi)}{\xi^{k+1}} d \xi
$$

где интегрирование ведется по окружности

$$
\Gamma=\left\{z \in \mathbb{C}:|z|=r, \rho<r<\frac{1}{\rho}\right\} .
$$

Это позволяет нам получить следуюшие оценки диагоналей оператора $A^{-1}$ :

$$
\left\|B_{k}\right\| \leqslant \frac{1}{2 \pi} \int_{\Gamma} \frac{\left\|\Phi_{A^{-1}}(\xi)\right\|}{\left|\xi^{k+1}\right|} d \xi \leqslant 2\left\|A^{-1}\right\| \rho^{|k|}, \quad k \neq 0, \quad\left\|B_{0}\right\| \leqslant\left\|A^{-1}\right\| .
$$

Из проведенных рассуждений следует теорема.

Teоpema 1. Пусть оператор $A$ из класса $\operatorname{End}(X, S, \gamma)$ обратим. Тогда обратный оператор $A^{-1}$ также имеет псевдоразреженную матрицу со следующим законом убивания әлементов $B_{i j}$ :

$$
\sup _{i-j=k}\left\|B_{i j}\right\| \leqslant 2\left\|A^{-1}\right\|\left(\frac{\gamma}{1-\sqrt[l]{\frac{2^{l+1} M\left\|A^{-1}\right\|(1-\gamma)^{l}}{(1-\gamma)^{l}+2^{l+1} M\left\|A^{-1}\right\|}}}\right)^{|k| / n_{l}}, \quad k \neq 0
$$

\section{СПИСОК ЦИТИРОВАННОЙ ЛИТЕРАТУРЫ}

[1] Блатов И. А. Об алгебрах операторов с псевдоразреженными матрицами и их приложениях // Сиб. матем. ж. 1996. Т. 37. №1. С. 36-59.

[2] Баскаков А. Г. Оценки элементов обратных матриц и спектральный анализ линейных операторов // Изв. РАН. Сер. матем. 1997. Т. 61. №6. С. 3-26.

[3] Demko S., Moss W. F., Smith P. W. Decay rates for inverses of band matrices // Math. Comp. 1984. V. 43. № 167. P. 491-499.

[4] Курбатов В. Г. Линейные дифференциально-разностные уравнения. Воронеж: Изд-во Воронежского ун-та, 1990.

[5] Шубин М. А. Псевдоразностные операторы и их функция Грина // Изв. АН СССР. Сер. матем. 1985. Т. 49. №3. С. $652-671$.

[6] Азарнова Т. В. Оценки элементов обратных матриц для некоторых классов линейных ограниченньх операторов // Изв. вузов. Матем. 1998. № 3. С. 74-77.

[7] Baskakov A. G., Asarnova T.V., Uskova N.B. On the structure properties of some classes of infinite matrices // Proceedings of the Tenth Crimean Autumn Mathematical School-Symposium "Spectral and Evolution Problems". Simferopol, 2000.

[8] Азарнова Т. В. Матричные методы оценки ядер обратных операторов для некоторых классов интегральных операторов // Труды III Международной конференции женщин-математиков. Вып. 1. Воронеж, 1995. С. 4-9.

[9] Азарнова Т.В., Колесников И.А. Достаточное условие обратимости интегральных операторов с медленно меняющимся ядром // Вестник фак-та ПММ. Вып. 2. Воронеж: Изд-во Воронежского ун-та, 2000. С. 3-12. 